\title{
Technical Note: Total Dissolved Gas Pressure (TDGP) Sensing in the Laboratory
}

\author{
Brian G. D'Aoust \\ Common Sensing Inc., Clark Fork, ID
}

Abstract

An increasing number of routine laboratory analyses and procedures require the use of gas-free distilled water with a reproducible level of TDGP (1). This proceeds from the need to avoid bubble formation when the water is heated or subjected to lowstrength ultrasonic fields. The use of a simple oxygen sensor $(2,3)$ to provide an estimate of the degree to which distilled water has been degassed can introduce inconsistent results due to different distillation methods, height above sea level, and conditions and duration of storage. As shown by Eaton et al. (4), the degree of deaeration and procedures used to affect it can be a major contributor to such errors in currently used apparatus. Since air is approximately $78 \%$ nitrogen and $21 \%$ oxygen, a direct measurement of TDGP will give a more complete picture and is obviously preferable. Two recent studies $(5,6)$ have demonstrated the importance of using a consistent TDGP level for reliable comparisons of the results of dissolution studies. The history, applications, and physical embodiment of the method are described.

\section{Introduction and Background}

T he purpose of this article is to briefly describe the function of a class of sensors and related instruments that are seeing increased use in dissolution technology and other laboratory environments needing quality control of gas-free water. A history of the chemical analysis of dissolved gases reveals that gravimetric analyses were initially the only means available, and therefore the units used expressed gas content (weight/volume) rather than gas pressure (7). As we know from Henry's Law, the molar gas content or concentration, $C$, of gas in a solution is proportional to its partial pressure in the atmosphere contacting the solution and a solubility coefficient, $\beta_{g}$, that is unique to each gas, solvent, and temperature (8):

$$
\mathrm{C}_{\mathrm{g}, \mathrm{T}}=\mathrm{P}_{\mathrm{g}} \beta_{\mathrm{g}}
$$

Even so, thermodynamic considerations establish that the chemical potential, $\mu$, of an ideal gas is directly related to its partial pressure by the standard equation

$$
\mu=R T \ln P+\mu^{0}
$$

in which $R$ is the gas constant, $T$ is Kelvin temperature, $P$ is the gas pressure, and $\mu^{0}$ is an integration constant dependent on the nature of the gas and the temperature (9). Yet the concept of dissolved gas pressure can introduce some degree of confusion for those used to thinking in gravimetric terms, notwithstanding the inherent simplicity of pressure measurements and the intuitive logic of measuring partial or total pressures directly. However, it is only recently that the available technology and instrumentation have made such measurements feasible for routine use.

Since the solubility coefficient for any gas in various solvents is unique and temperature-dependent (10), if one measures, at two different temperatures, the TDGP of a solution that is in equilibrium with one atmosphere of air pressure $(760 \mathrm{~mm} \mathrm{Hg}, 29.92 \mathrm{in.} \mathrm{Hg}, 1013.25$ millibar), the same pressure will be indicated, but the content or molarity of each constituent gas represented by that pressure will increase or

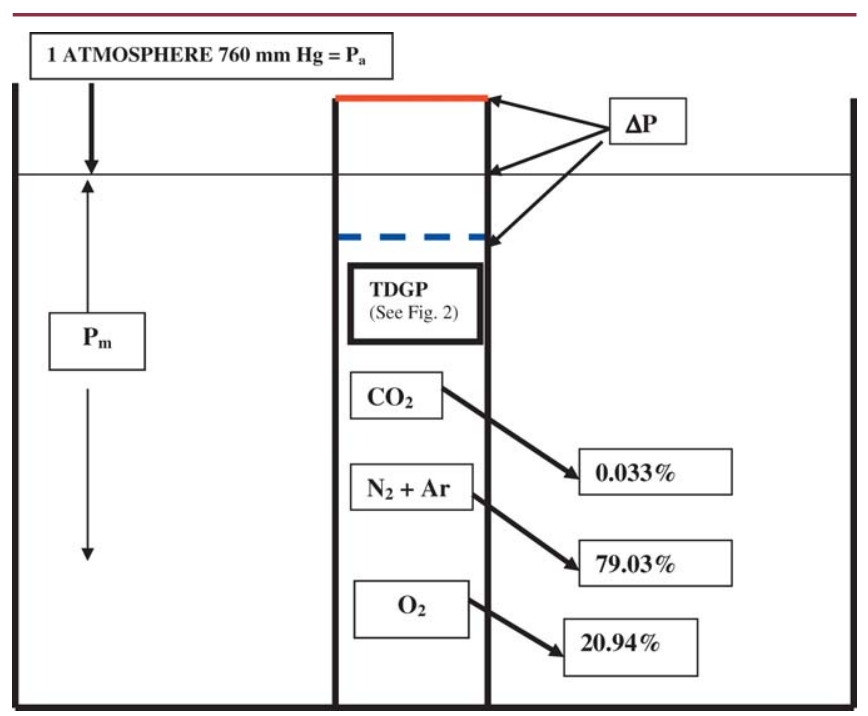

Figure 1. Analytical opportunities provided by direct TDGP sensing.

decrease according to whether the temperature is lower or higher, respectively. At equilibrium at either temperature, one can say the degree of saturation is $100 \%$ of one atmosphere, and at equilibrium, the TDGP measurement will be the same at each temperature. Thus the TDGP, or $P_{\mathrm{T}}$, equals the sum of the partial pressures of all constituent dissolved gases as well as the solvent vapor. Or, for solutions that have been equilibrated with air:

$$
\mathrm{P}_{\mathrm{T}}=\mathrm{p}_{\mathrm{H} 2 \mathrm{O}}+\mathrm{p}_{\mathrm{O} 2}+\mathrm{p}_{\mathrm{N} 2}+\mathrm{p}_{\mathrm{Ar}}+\mathrm{p}_{\mathrm{CO} 2}+\mathrm{p}_{\mathrm{n} 1}+\mathrm{p}_{\mathrm{n} 2}+\ldots+\mathrm{p}_{\mathrm{ni}}
$$

where $\mathrm{n} 1, \mathrm{n} 2$, and ni are all other trace gases.

This is shown in Figure 1, which diagrams the relative percentage content of the constituent gases in air. The surface atmospheric pressure $P_{\mathrm{a}}$, which at equilibrium dictates the concentration of gases, is represented at the top of the diagram as one atmosphere. The total mechanical or hydrostatic pressure, $P_{\mathrm{m}}$, will be equal to

$$
P_{\mathrm{a}}+(Z \times 22.35 \mathrm{~mm} \mathrm{Hg} / \mathrm{ft})
$$




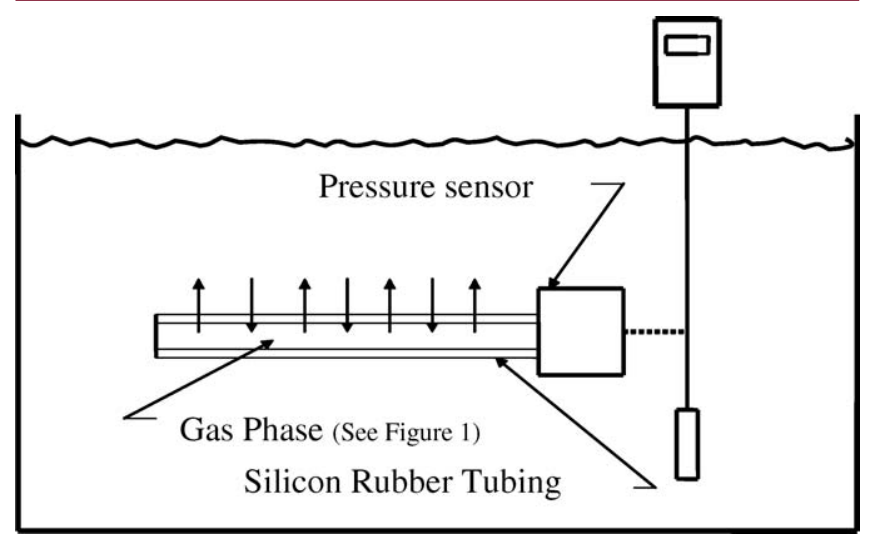

Figure 2. Schematic representation of TDGP measurement.

where $Z$ is the depth of a water column in feet. The red line at the top symbolizes a supersaturated condition. The sum of $P_{\mathrm{a}}$ and $P_{\mathrm{m}}$ must always exceed $P_{\mathrm{T}}$ when measuring supersaturated levels of TDGP. The dashed blue line beneath the surface indicates an undersaturated condition, which deaeration techniques strive to achieve (1).

As shown in Figure 1,99\% of atmospheric gases are represented by $\mathrm{N}_{2}, \mathrm{O}_{2}, \mathrm{Ar}$, and $\mathrm{CO}_{2}$. Water vapor pressure, on the other hand, will be dependent on temperature and, in any gas phase in equilibrium with the water, will be saturated or $100 \%$ relative humidity $(\mathrm{RH})$. In a situation where there is complete gas extraction (difficult to achieve), only water vapor pressure would be measured - at $20^{\circ} \mathrm{C}$ at about $17 \mathrm{~mm} \mathrm{Hg}$.

If the solution is heated or cooled, the solubility of each gas will either decrease or increase, respectively, and provided there is no gas exchange external to the solution, the partial pressures of each gas will increase or decrease in the opposite direction to solubility leading to supersaturation or undersaturation, shown by the red or blue line, respectively, in Figure 1. There will be a net flux driven by the resulting gradients in partial pressures until equilibrium is re-established.

As illustrated in Figure 1, in a hypothetical container surrounding the liquid system in which gas exchange is prevented, the TDGP will immediately reflect any changes in temperature because of its effect on solubility (10).

\section{Basis of the Method}

Consider, then, a small gas phase bounded by a gaspermeable membrane suspended in solution; at equilibrium (our required conditions of measurement) there is no net flux of any gas into or out of the gas phase, because there is no gradient in the concentration or partial pressure of each gas between the gas phase or the solution.

With today's accurate pressure sensors in combination with the permeability of small diameter dimethyl silicon tubing, the measurement of TDGP in a small gas phase is now possible.

\section{History}

The history of the method is an interesting example of how different fields of research intersect. The first simple

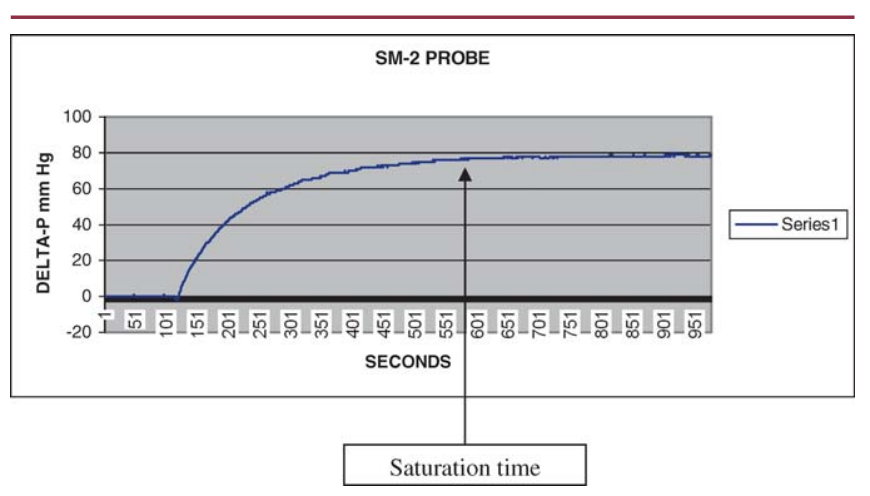

Figure 3. SM-2 probe saturation time.

device constructed for the direct measurement of Total Dissolved Gas Pressure (TDGP) using a silicon rubber (dimethyl silicon) gas-permeable membrane was developed by Dr. Ray Weiss (11) of Scripps Institute of Oceanography in 1971. This was in response to an increasing need for a more rapid technique for measuring gases in natural waters. The need itself arose from a chronic problem in many impounded rivers, such as the Columbia and Snake Rivers in the northwestern United States, in which spilling at dams as well as seasonal temperature increases caused supersaturation, which at sufficient levels could be lethal to fish due to bubble formation in their tissues (12).

Up to that time, water sampling, storage, and extraction and analysis - usually by gas chromatography — was the preferred method. Though accurate and capable of multiple gas analysis, the method was tedious, time consuming, and fraught with storage errors.

Weiss' original device was a hand-held frame holding a considerable length of silicon rubber tubing and a simple oil-filled dial pressure gauge. Because it was a gauge sensor, it only provided a read-out as "DELTA- $\mathrm{P}$ " or $\Delta \mathrm{P}$, the difference between the TDGP and atmospheric pressure rather than the absolute TDGP pressure, in other words, the absolute value of the differences shown by the red line or blue line in Figure 1. While direct and more rapid than field sampling followed by lab analysis, the unit was also cumbersome and slow and could only be used at the surface of the water. What was needed was a probe configuration that could be placed at the end of a cable for measurements at depth. At the time, few electronic pressure sensors had built-in signal conditioning, and different cable lengths required separate calibrations.

In 1971 Texas Instruments introduced integrated-circuit pressure sensors using laser trimming of on-board compensating resistors to minimize temperature coefficients and provide a 0-5 volt output to match industry standards. One version provided an internal vacuum reference so that a true absolute pressure guage could be realized in the same integrated chip package. This advance presented the opportunity for simple integration into a compact, watertight probe, which was introduced by D'Aoust et al. in 1975. Figure 2 shows the essentials of the method (12); it could be connected to a cable of any length and provided a conve- 


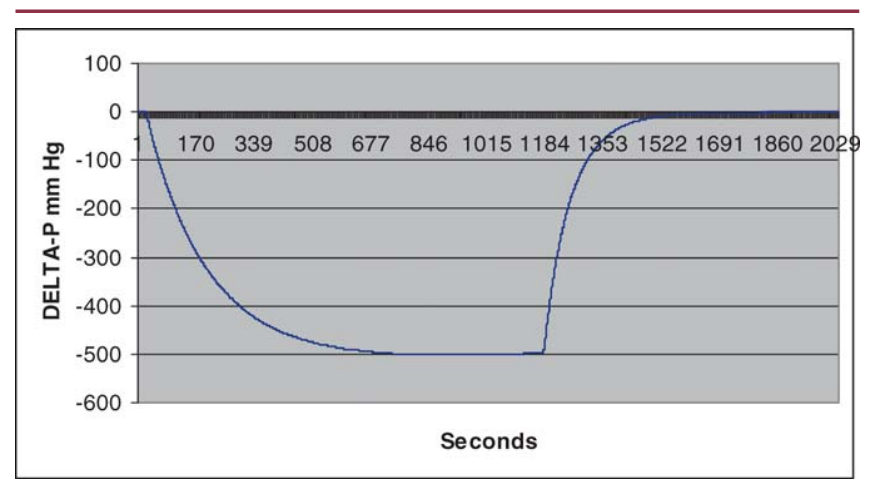

Figure 4. FDA modified SM-1(3) probe measurement of degassed water with some stirring.

nient means to track this critical parameter at remote sites $(13,14)$.

The evolution of this instrumentation has proceeded along a path reflecting the needs of aquaculture and environmental monitoring. This has resulted in increasingly integrated instruments providing many of the relevant parameters for the analysis of dissolved gases discussed above, such as barometric pressure, $\mathrm{pO}_{2}, \mathrm{TDGP}-\mathrm{pO}_{2}$ (approximates $\mathrm{N}_{2}+\mathrm{Ar}$ ), $\Delta \mathrm{P}\left(=\mathrm{TDGP}-\mathrm{P}_{\mathrm{a}}\right)$, \% saturation of TDGP, and \% saturation of $\mathrm{O}_{2}$. Microprocessors have now been added so that these "derived" parameters can be calculated from the primary sensor outputs and the data logged or downloaded directly or by telemetry in accordance with current standards.

\section{Measurements}

Since the method depends on the filling of a small but finite gas phase, a finite time must therefore elapse before an equilibrium measurement can be made. Figure 3 indicates a typical saturation curve for a sensor when measuring supersaturation while gently stirring. A measurement of the opposite condition, undersaturation, is shown in Figure 4, which shows an approach to an equilibrium measurement in deaerated water.

As is evident from Figures 3 and 4, the elapsed time required for an accurate measurement can present a potential error if the required sample time is not allowed. This has generated efforts to optimize the kinetics of the probe by searching for membranes with high gas permeability, increasing the surface-area-to-volume ratio of the sensor, decreasing the thickness of the membrane wall, and eliminating the "residual" gas volume of the pressure sensor itself, that is, the gas volume not directly under the membrane surface area.

As in all engineering problems, the compromises inherent in these approaches involve a choice among the features that are considered to be the most desirable characteristics of the TDGP sensor; different applications will affect such choices. Cost, size, stability, required sample time, ruggedness of design, ease of replacement, required maintenance, need for recalibration, and type of electrical output are among the characteristics that need to be considered in arriving at the best design. A sensor that is adequate for routine laboratory use may be inadequate for field or industrial deployment where labor costs tend to be critical. On the other hand, some highly specialized applications, such as oceanographic studies of sea-surface gas exchange (15), have led to systems optimized for sample time at the expense of high cost, bulk, and therefore unsuitability for routine laboratory analysis.

Most TDGP sensors now available require a sample time of 4-5 minutes at $20.0^{\circ} \mathrm{C}$, slower at lower temperatures. It follows that agitation of the probe or otherwise arranging for adequate velocity of the sample water past the membrane in a flow-through housing is desirable for on-line systems and will minimize the required sample time.

The author's own experience in the field has revealed that a perception of limited markets has been the main factor in limiting motivation for development of a dedicated and integrated dissolved gas pressure sensor by high-tech companies capable of doing so, in which modern production techniques are brought to bear on the required specifications. However, as more applications are discovered, this may be something that we can look forward to in the future.

\section{Precautions}

\section{Bubble Formation.}

In measuring supersaturation (Figure 3), care must be taken that the total hydrostatic pressure $P_{\mathrm{a}}+P_{\mathrm{m}}$ (Figure 1) in the water where the TDGP probe is situated equals, or preferably slightly exceeds, the TDGP being measured. If $P_{\mathrm{a}}+$ $P_{\mathrm{m}}<\mathrm{TDGP}$, bubbles can form, and since they preferentially "stick" to silicon rubber membranes, this situation can lead to a higher steady-state equilibrium TDGP because there is now a three-compartment system being measured that depends on a number of variables. Even if a steady state can be achieved in such a situation, it will not reveal the true TDGP. Flow-through systems are therefore preferred in supersaturated systems since a flow-related excess pressure at the sensor membrane surface will occur. However, in such a setup, checks for bubbles should be made whenever the system is depressurized and repressurized; accordingly, gas pressure relief ports should be included in the design of such apparatus.

\section{Long-Term Immersion of Probes}

Although silicon rubber tubing has been found to be a very convenient membrane for the separation of the dissolved gas into the gas phase, environmental applications (11) of the method have revealed a need for periodic drying of the sensor if immersion periods longer than two weeks are required, particularly when silicon rubber is the membrane material used. This is because condensed water can slowly accumulate inside the lumen of the tubing even though temperature is constant, and with enough time, cross-bridging of the water droplets can cause errors in the 
pressure reading due to meniscal forces. The phenomenon is related to "Soret distillation"(16), which has been shown to allow distillation of water at lower temperatures across an organic polymer matrix.

\section{Conclusions}

Direct measurement of TDGP with newly available sensors that can be calibrated to read out in any desired pressure units provides a simple, reliable means of quality control in any laboratory application requiring gas-free or partially degassed water or other solvents. The advent of SOIC technology, highly reliable and accurate pressure sensors, and highly permeable polymer membranes has allowed integration of these different technologies to further streamline design and lower costs.

\section{References}

1. Moore, T. W. Dissolution Testing: a fast efficient procedure for degassing dissolution medium. Dissolution Technol. 1996, 3 (2), 3-5.

2. Curley, T.; Forsyth, R.; Sun, S.; Flizar, K.; Colletto, M.; Martin, G. P. Measurement of dissolved oxygen as a determination of media equilibrium during dissolution testing. Dissolution Technol. 2004, 11 (4), 6-11.

3. Diebold, S. M.; Dressman, J. B. Dissolved Oxygen as a Measure for De- and Reaeration of Aqueous Media for Dissolution Testing. Dissolution Technol. 1998, 5 (3), 13-16.

4. Eaton, J.; Deng, G.; Hauck, W. W.; Brown, W.; Manning, R. G.; Wahab, S. Perturbation Study of Dissolution Apparatus Variables-A Design of Experiment Approach. Dissolution Technol. 2007, 14 (1), 20-21.

5. Nithyanandan, P.; Deng, G.; Brown, W.; Manning, R.; Wahab, S. Evaluation of the Sensitivity of USP Prednisone Tablets to Dissolved Gas in the Dissolution
Medium Using USP Apparatus 2. Dissolution Technol. 2006, 13 (3), 15-18.

6. Gao, Z.; Moore, T. W.; Doub, W. H.; Westenberger, B. J.; Buhse, L. F. Effects of deaeration methods on dissolution testing in aqueous media: A study using a total dissolved gas pressure meter. J.Pharm. Sci. 2006, 95, 1606-1613.

7. Winkler, L. W. Die Bestimmung des im Wasser gelasten Sauerstoffes. Chem. Ber. 1888, 21, 2843-2855.

8. Glasstone, S. Textbook of Physical Chemistry, 2nd ed.; Van Nostrand: New York, 1946; Chapter 10.

9. Glasstone, S. Textbook of Physical Chemistry, 2nd ed.; Van Nostrand: New York, 1946; Chapter 4.

10. Weiss, R. F. The solubility of nitrogen argon and oxygen in water and seawater. Deep Sea Res. 1970, 17, 721-735.

11. Weiss, R. F. Permeable membrane gas saturometer. U.S. Patent 3,871,228, March 18, 1975.

12. D'Aoust, B. G.;White, R.; Siebold, H. Direct Measurement of Total Dissolved Gas Pressure. Undersea Biomed. Res. 1975, 2, 141-149.

13. D'Aoust, B. G.; Clark, M. J. R. Analysis of Supersaturated Air in Natural Waters and Reservoirs. Trans. Amer. Fisheries Soc. 1980, 109 (6), 708-724.

14. D'Aoust, B. G. Field Service and Instrument Performance During the 1995 Season Monitoring Air Saturation in the Columbia and Snake Rivers. U.S. Army Corps of Engineers Contract DACW57-93-C-0015 and Bureau of Reclamation 1425-3-PE-17-01390, 1995.

15. McNeil, C. L.; Johnson, B. D.; Farmer, D. M. In Situ Measurement of Dissolved Nitrogen and Oxygen in the Ocean. Deep Sea Res. 1995, 17, 721-735.

16. Hammel, H. T.; Maggert, J. E. Method and apparatus for separating solvent from solute. U.S. Patent 4,153,546, May 8, 1979. 